\title{
Innovating in the medical device industry - challenges \& opportunities ESB 2015 translational research symposium
}

\author{
Y. Bayon $\mathbb{1}^{1} \cdot$ M. Bohner ${ }^{2}$ D. Eglin ${ }^{3} \cdot$ P. Procter $^{4} \cdot$ R.G. Richards ${ }^{3} \cdot$ J. Weber $^{5} \cdot$ \\ D.I. Zeugolis ${ }^{6,7}$
}

Received: 31 March 2016 / Accepted: 27 July 2016 / Published online: 23 August 2016

(C) Springer Science+Business Media New York 2016

\begin{abstract}
The European Society for Biomaterials 2015 Translational Research Symposium focused on 'Innovating in the Medical Device Industry - Challenges \& Opportunities' from different perspectives, i.e., from a non-profit research organisation to a syndicate of small and mediumsized companies and large companies. Lecturers from regulatory consultants, industry and research institutions described the innovation process and regulatory processes (e.g., 510K, PMA, combination product) towards market approval. The aim of the present article is to summarise and explain the main statements made during the symposium, in terms of challenges and opportunities for medical device industries, in a constantly changing customer and regulatory environment.
\end{abstract}

Y. Bayon

yves.bayon@covidien.com

1 Medtronic - Sofradim Production, 116 Avenue du Formans, Trévoux 01600, France

2 Dr Robert Mathys Foundation, Bischmattstr. 12, Bettlach CH2544, Switzerland

3 AO Research Institute Davos, Clavadelerstrasse 8, Davos 7270, Switzerland

4 Medical Device Industry Consultant, Divonne les Bains 01220, France

5 Boston Scientific, Martinolaan 50, Maastricht 6229GS, Netherlands

6 Regenerative, Modular \& Developmental Engineering Laboratory (REMODEL), National University of Ireland Galway (NUI Galway), Galway, Ireland

7 Centre for Research in Medical Devices (CÚRAM), National University of Ireland Galway (NUI Galway), Galway, Ireland
Innovation and new product development in the medical device industry have largely been technology driven in the last decades but always to solve clinical needs. Advancements have been often rapid and significant. Today's biomaterial implants have reached high standards; consequently, the state of art treatment generally supports acceptable return to daily activities and quality of life. Nonetheless, today's product development occurs in a complex healthcare environment, with multiple stakeholders involved. Each of these stakeholders can have different and sometimes conflicting requirements and expectations. While technological innovation to solve current clinical needs may still be the driving force behind new product development, in many cases it is not sufficient to reach the clinic.

Innovation in the medical device industry can take many forms. From an entrepreneurship perspective, it does not require inventing something completely new; it can simply involve applying an existing idea in a new way or to a new situation. Innovation includes (i) rapid cadence of market release of new devices or services; (ii) scientific communication on a company's latest products with key opinion leaders support; (iii) clinical education of care providers; (iv) skilled communication channels; and (v) brand equity or the value of the brand name. Those are the five pillars of success in medical devices business. Innovation is greatly influenced by the patients, the care providers, the physicians, the payers, the policymakers and the producers who can all influence real-world healthcare decision-making. Putting on the market innovative products also depends on the fulfillment of regulatory requirements (which vary from continent to continent and even between countries), which are difficult but are in place to attempt to guarantee the safety and efficacy of any market approved products. This is a crucial bottleneck in time and cost for a biomaterial-based device and therapy. 
This topic was specifically addressed at the European Society for Biomaterials 2015 in Krakow, where the Translational Research Symposium focused on 'Innovating in the Medical Device Industry - Challenges \& Opportunities' from different perspectives. This was from a nonprofit translational research organisation to a syndicate of small and medium-sized companies (SMEs) and large companies. Lecturers from regulatory consultants, industry and research institutions described the innovation process and regulatory processes (e.g., 510K, Pre-market approval [PMA], combination product) towards market approval. The customer and the constantly changing regulatory environment represented both innovation challenges and opportunities for medical device industries.

\section{Medical translational research: a different route to fundamental research}

The AO Research Institute Davos has nearly 60 years of experience in translation of innovative ideas to solve clinical problems of the musculoskeletal system improving surgical techniques for patient care bringing the patient back to mobility and work (http://www.medicaldevicedevelopments.com/features/featurereforming-europeanmedical-technology-regulation/, Accessed on 15 March 2016). The patents awarded by this organisation were held by the AO Foundation and either licensed, generating royalty fees, or sold to industry and invested back into an endowment of the AO Foundation, which funds a large amount of the research. Despite this long-term effective record in translation, the successful way from idea to product has become much more arduous and complex. In the field of osteosynthesis, this is partly due to the wide range of proven products covering clinician needs available today, along with regulatory hurdles, and the more and more risk adverse/conservative nature of manufacturers towards innovative technology. Thus, novel products can often generate incremental benefit at best, in comparison to established products. Innovative concepts for fracture fixation departing from the classical osteosynthesis devices exist. They are moving towards faster repair along with treatment of major co-morbidities such as infection. They are driven by scientific innovation in biomaterials and biologics. However, the economics of the field are driven downwards by many factors including increasing medical approval hurdles (e.g., clinical benefit, manufacturing cost, selling price, market approval) and associated new product development costs. The policymakers, healthcare providers, insurance companies and patient groups also add their demands to the demands and wishes of surgeons and device producers. Therefore, massive resources are required from an institution aiming to perform translational research, beyond what was required in the past. As soon as a biomaterial is considered as a delivery device, approval costs become inhibitory to innovation for the majority.

\subsection{From the biomaterials laboratory to the preclinical facilities}

For the preclinical side, accreditation of $\mathrm{R} \& \mathrm{D}$ processes and facilities is important with certifications such as ISO (International Standards), GLP (Good Laboratory Practice embodies a set of principles that provides a framework within which studies are planned, performed, monitored, recorded, reported and archived) and AAALAC (AAALAC International is a private, non-profit organisation that promotes the humane treatment of animals in science through voluntary accreditation and assessment programs) for a preclinical facility. The team must have a mix of academic and more development-oriented members in various fields in order to ensure relevance of the performed studies when presenting them to notified bodies. In medical translational research, it is extremely important to know very early on, i.e., before the start of large investments in preclinical research, if the idea is really novel; what is the current patent situation for this area throughout the world; what is the current situation on the market; what is the current clinical solution (or workaround); what are the regulatory hurdles to such a solution (regionally they may vary); what is the financial cost and market for such a clinical problem (regional and world-wide). Based upon this, the estimated R\&D costs associated to preclinical work can be put in perspective and a decision on the worthwhile investment taken.

Once you have performed such searches and intend to protect and follow the idea, the project and the preclinical work need to be differentiated from fundamental research projects. PhDs and postdocs need to publish for their careers while a real medical translational research project needs to be kept confidential at minimum until patent protection is submitted. This can delay academic recognition, yet helps make the idea tradable after filing the patent. Since patent protection is not cheap, initial studies to test the hypothesis are recommended before patent filing, but must not be made public and therefore need to be under the project lead of Research Associates with technician support (whose careers are not based only upon academic publications). An alternative is to patent locally (one country) first giving 1 year protection, since the first to invent system has been replaced with the first to file a patent system in 2013. This allows the inventor time to work and see if the idea is worth to take to the next level of patent at the international level.

\subsection{Steps beyond a successful preclinical study}

The AO Research Institute Davos has moved in the 90's towards biology, tissue engineering and regenerative medicine, because of an increasing clinical interest/demand for 
the targeted and controlled delivery of active molecules (or cells) to specific parts of the body that requires extremely well-characterised biomaterial delivery platforms. Examples include the localised delivery of antibiotics to infection sites or the release of stimulants for local tissue regeneration [1]. The biomaterials ideally should be biocompatible, biodegradable and allow controlled temporal release of the active substances. This involves a lot of developmental controls and after proof of concept at the preclinical research level, costs and legal hurdles for the jump from this to get a product to the bedside are enormous. What should one insource and what should one outsource? [2, 3]

Many orthopaedic companies no longer will buy a proof of concept, especially when the manufacturing and regulatory hurdles are as high as for combination products. Thus, alternative funding concepts and processes are needed. Technology transfer and licencing to industry, certification (e.g., CE), regulatory issues, GLP and GLM for tissue engineering are all areas that have to be considered for this question. Despite the improved professional set up to nurture, approve and expose medical translation projects in research institutions, key areas of the translation cycle required to move scientific biomaterials innovation to an industrial environment are increasingly challenging and uncertain. It is obvious that today the steps from bench to bedside have become exponentially more difficult than the devices of the past.

\section{A clinical need-based biomaterial strategy "reducing the risk that innovation" is "lost in translation"}

\subsection{Status quo}

The gap between unmet clinical needs and biomaterials qualified to meet these needs is widening. It is increasingly evident that orthopaedic clinicians are becoming reluctant to use what they consider clinically unproven so-called "new" biomaterials products [4]. The orthopaedic industry itself has not made a success of advanced biomaterials, such as osteogenic proteins. Neither the bone morphogenetic protein BMP-2 nor BMP-7 has fully delivered the hope for revolution in the bone healing. There are numerous law suits due to "off-label" use, and safety and efficacy concerns continue to limit BMP use. These high-end biomaterials are now being phased out. Over the same period there has been a decline in innovation in the orthopaedic industry. This is a direct consequence of the US Department of Justice's investigation of the Orthopaedic Industry and the industry wide subsequent enforcement of compliance: Chatterji and Fabrizio [5] stated that "we find evidence of dramatic decline in the rate of innovation at the firm and industry level". The larger medical device companies are increasingly focused on acquisition of biomaterials rather than developing their own products and the last 5 years has seen a number of acquisitions such as Orthovita (United States) by Stryker (United States) (2011), Biomimetic Therapeutics by Wright Medical (United States) (2013) and Etex (United States) by Zimmer (United States) (2014). There are now few remaining independent biomaterials makers of any stature. One example is DSM (The Netherland), who acquired Kensey Nash (United States) and Skeletal Kinetics (United States). The commercial resources necessary to qualify new and innovative biomaterials are now scarce. The biomaterials developers themselves are often caught in an innovation paradox. If a biomaterial is not novel (e.g., has the same claims as predicated biomaterials), it will not attract investment, if it is novel (e.g., needs a new claim approval) it will also not attract investment. In any case without prospective clinical studies that prove a measurable benefit both reimbursement agencies and hospitals are increasingly reluctant to accept "novel" biomaterials. So with all these factors combined, it becomes more likely that innovation will not make it through to an end product as investors/developers seek to minimise project risks. So what strategy could enable innovation in biomaterials?

\subsection{A clinical need lead biomaterials strategy: Many biomaterials originated from a technology lead approach}

In this approach an available technology is adapted to fit a broader and broader range of user needs, eventually risking going "off-label". In the authors' opinion this is largely what happened with some branded calcium phosphate (CaP) cements. In 30 years since CaP's were introduced, there have been hundreds of $\mathrm{CaP}$ projects (authors' estimate) all aiming to be non-load bearing void fillers. Less than 10 of these products make up $80 \%$ of the market volume today and only two of these are available with enhanced claims (antibiotic, screw augmentation in EU markets only). Yet is clear from the off-label use reported for $\mathrm{CaP}$ cements that there are clinically unmet needs. Examples of these unmet needs are: load sharing/bearing applications, adding bone quality enhancement, or infection control measures via drugs. The authors foresee that the following will increase the success rate of translation of innovative biomaterials projects into clinically valued treatments:

1) An evidence-based definition of the unmet clinical needs and the corresponding clinical outcomes;

2) Assessment of reimbursement potential vs. the evidence that clinical outcomes are improved; 
3) Select technologies, intraoperative handling and clinical site delivery appropriate to the unmet needs;

4) Develop biomaterial properties appropriate to meet specific indication needs (e.g., avascular necrosis risk).

\subsection{The novel bone augmentation solutions syndicate (BASS) commercialisation model}

\subsubsection{The first BASS project}

In 2014 one of the authors presented the BASS concept [6] as a novel commercial pathway to bridge the gap between the research and commercial environments. The underlying motivation is that there are significant unmet needs and drivers in the field of screw implant fixation in poor quality bone. To commercially realise a hybrid approach, where a biomaterial is used in combination with an implant the author has grouped companies around collaborative projects. The first BASS project illustrates this approach as follows: it is the combination of an implantable screw that enables cement augmentation (Innovision Inc., United States) with a novel $\mathrm{CaP}$ cement that allows stop-start behaviour (InnoTERE GmbH D) with an optimised mixing and delivery system (Medmix AG $\mathrm{CH}$ ) into a clinically attractive package (PBC Ltd). After successfully completing cadaver workshops augmenting screw fixation in femoral neck and tibial fractures the N-Force cement delivery/fixation screw, and the iN3 cement pack (cement, gun, static mixers and cannulae) were both $\mathrm{CE}$ marked by a commercial entity (Celgentek Ltd, Ireland) and have now entered the European marketplace. The unmet needs that were addressed (vs. current $\mathrm{CaP}$ cements) were ease of intraoperative mixing and delivery that saves time, and start-stop behaviour that saves both time and cost (vs. opening a second cement package to a second augmentation in the same procedure).

\subsubsection{The second BASS project}

The second BASS project is the development of injectable bone void filler with the potential to minimise vascular impact in a compromised vascular environment. This unmet need resulted from the post-market surveillance observation that $\mathrm{CaP}$ cements compromised the vascular recovery in femoral neck fractures [7]. The root cause of the vascular compromise was suggested to be the presence of $\mathrm{CaP}$ cement in the fracture line. A later research project showed that $\mathrm{CaP}$ nanoparticulates dispersed in a hyaluronic acid hydrogel provided surprisingly rapid new bone formation [8]. (It was actually the control in a study where the test material additionally contained zoledronic acid). It is suggested that the $\mathrm{CaP}$ content can be increased to the level that is detectable with a screw pull-out test in bone (preliminary tests suggest $30-40 \%$ ). If this proves to be feasible then a new (to orthopaedic use) injectable cement may be developed that would combine primary stabilisation at surgery, and rapid new bone formation (compared to bulk $\mathrm{CaP}$ cements). Such a combination material may well be suited to an avascular risk environment, such as proximal humeral and femoral neck fractures.

\subsection{Concluding comments}

Over the last years, innovation in orthopaedic biomaterials has slowed down and, in the authors' opinion, is unlikely to accelerate unless there is a greater focus on unmet clinical needs and the production of clinical evidence that innovation is worth the investment. Biomaterials developers favour "me too" products as the regulatory pathway is less onerous, while a novel claim is very desirable but a much higher investment risk. So innovation often fails to translate from successful laboratory scale proof of concept to commercial success and to establish subsequent clinical evidence that sustains reimbursement. A quite different kind of biomaterials product strategy will be required, if the gap between unmet clinical needs and biomaterials qualified to meet these needs is narrowed. The biomaterials that are most likely to enable this are those capable of generating quantitative clinical evidence of efficacy in specific indications.

\section{Insights into innovation and collaboration for early stage medical device development - The large medical device company perspective}

\subsection{Technological problem}

Innovation aims at solving problems, but which ones from an industry perspective. The Medical Device industry probably encompasses most, if not all, of the technology fields including (bio)chemistry, (bio)physics, biology, medicine, applied mathematics and computation, and of course manufacturing technology. In addition, industry standards are and must be extremely high for the safety of patients.

Surgeons implant devices made of metals and polymers that need to last for a lifetime in a very hostile environment, the human body, in terms of (bio)chemical attacks and (bio) mechanical stresses. Batteries and electronic systems are also implanted and they should last for up to 15 years with an extremely low failure rate, generally lower than $0.01 \%$. Sensors are more and more incorporated in medical devices, 
for the interrogation and/or monitoring of a large range of physical parameters, requiring fast numerical analysis and user friendly displays. Sensitive pharmaceuticals drugs are also integrated in medical devices, the combination devices. All these solutions should not be designed by integrating the sole technical/scientific dimension, but should include many other aspects of medical device commercial translation, such as logistics, inventory control, tracking, counterfeiting, regulatory requirements. As a general rule, in pharma/biotech, one technology project out of ten makes it to a product that goes to the clinical trial phase.

\subsection{Clinical trial problem}

For new kinds of devices, such as for PMA regulated in the United States, the biggest hurdle can come at the very end with the clinical trials. One never knows if it really works until clinical trials have been done at significant financial and time costs. Whenever possible, the failure risk of product development at the clinical stage should be reduced by rigorous and robust preclinical evaluation, using the most appropriate model to be selected among the ones reported in the literature or to be specifically developed when required. They are still innovation needs for better preclinical models, not necessarily in terms of mimicking the clinical disease conditions, but more in terms of better prediction of the behaviour and performance of the devices, in the clinics [9]. The clinical development of devices can also fail and/or be very expensive. Sometimes, this may be explained by poorly designed clinical trials, e.g., due to inappropriate primary clinical outcomes and/or evaluation, lack of intermediate endpoints, insufficiently defined inclusion and/or exclusion criteria.

\subsection{Reimbursement pressure (\& Competition)}

The development of innovative solutions should meet considerable challenges, ever increasing cost of new product and pricing pressure from healthcare insurance companies. The trend of healthcare spending in OECD countries sharply slowed down in 2010 with the global economy crisis and bounced back since at lower annual rates than the pre-2010 time period (http://www.oecd.org/health/healthsystems/Focus-Health-Spending-2015.pdf. Accessed on 4 February 2016). In the same time, according to AdvaMed (Advanced Medical Technology Association, a US medical device trade association), the average price paid for medical devices dropped down in the United States, e.g., by $34 \%$ for drug eluted stents from 2007 to 2011. This can be explained by the reimbursement pressure, but also by other factors such as fierce competition (http://marketrealist. com/2013/09/must-know-device-prices-dropping-suggestsstrong-market/. Accessed 5 February 2016).
It becomes more and more important to develop valuefor-money propositions for payer acceptance, for any medical devices. Reimbursement pressure and economic hard times both strongly push in the same direction, doing more with less. Medical device companies do not like, but need, at the end, inexpensive - or affordable - and robust solutions.

\subsection{Expanding portfolio problem}

As the life expectancy is still improving, particularly at a fast pace in emerging countries, it means that an increasing number of diseases or malfunctions will have to be potentially treated during the lifetime of anyone. In particular, the Medical Device industry just creates new challenges, each time after the successful launch of new solutions. It will not stop soon.

For example, technology fields are emerging more and more strongly:

- Personalised medicine: today, patients suffer or die from postoperative adverse reactions. Tomorrow, affordable prognostic and/or diagnostic solutions may prevent such complications by the development and deployment of personalised medicine approaches.

- Regenerative medicine and advanced therapy medicinal products: after the first waves of disillusions, the regenerative medicine industry seems to reach a mature stage with upcoming cytotherapy solutions combined or not with biomaterials. The global regenerative medicine market is still evaluated as a potential huge market, dozens of billions US dollars.

- 3D printers: this technology should allow new designs and biomaterial combinations, which may lead to personalised biomaterials solutions fitting the specific needs of anyone.

- New biomaterials: top-down and bottom-up recent innovations should lead to materials with new and unique functionalities. For example, the trend goes with biodegradable solutions, not just synthetic polymers/ biopolymers, but also biodegradable batteries, sensors...

- Internet of Thing: this technology should support the continuous monitoring of anyone's condition.

Another push should be the expansion of products for lifetime treatments. Currently, a significant part of healthcare is spent during the last year of life. About $30 \%$ of Medicare expenditures are attributable to the small part of beneficiaries who die each year (https://www.cms. gov/Research-Statistics-Data-and-Systems/Research/Actuar ialStudies/downloads/last_year_of_life.pdf. Accessed 5 February 2016). Annual per capita healthcare costs are flat during the first part of lifetime and start to sharply increase from the age of 60, particularly in the United States (http:// 
ritholtz.com/2013/01/chart-of-the-day-health-care-spendingby-age-and-country/. Accessed 5 February 2016). The current practice is still predominantly to treat when something is seriously wrong. The future practice should be to treat in advance as well, supported by cost savings value propositions. This may lead to another dozens of thousands medical device solutions, some of them relying on biomaterials.

\subsection{Insights in innovation in the medical device industry - the reality}

It requires more often now a substantial investment in technological development to develop products outside boundaries, whereby a tiny fraction - low percentage range - of projects actually will be successfully launched in the market and that of those, only very few will be a blockbuster.

The current entrepreneurial landscape in Medical Devices is essentially made of SMEs. For example, Europe hosts about 25,000 medical technology companies of which SMEs, making up almost $95 \%$ of this industry, employ less than 50 people (small and micro-sized companies) (http:// www.medtecheurope.org/sites/default/files/resource_items/ files/MEDTECH_FactFigures_ONLINE3.pdf. Accessed 15 February 2016). And early stage innovation is primarily done at an SME level, generally focussing on single products with high-risk technological development (http://dspace. mit.edu/bitstream/handle/1721.1/2183/SWP-1930-18388674. pdf?sequence=1. Accessed 15 February 2016) [10]. Generally, quality aspects are at a low standard and very low mostly none - efforts have been done on manufacturing optimisation, this generating high to very high cost of goods sold (COGS; direct costs attributable to the production of the goods sold by a company). The disruptive technology/ product innovations of SMEs are potential acquisition targets by large companies, which will adapt the innovation to make products with ease of use, reliability and cost-effective manufacturing. These products may be further processed by upgrading the acquired technology, by developing line extensions and second generations by incremental innovation.

\section{Concluding remarks}

Innovation out of commercial boundaries should be fostered by collaboration, both with customers - e.g., patients, surgeons - and technology providers - e.g., universities, SMEs, large companies. The customers should share the needs in existing and new clinical areas and, the technology providers should translate these needs into commercially viable solutions, using existing or emerging developments.
The commercial translation is not exclusively driven by the technology itself, but complementary and mandatory skills such as industrialisation to cost-effective solutions, customer compliance (e.g., ease of use, reliability), clinical evidence, regulatory filing, marketing. It is therefore important to buildup appropriate networks such as the BASS.

Commercial translation can also be very expensive. As an example, Kyphon Inc, now incorporated into Medtronic, was created in 1994 for the development of an improved vertebroplasty treatment of spinal fractures by kyphoplasty. This minimally invasive surgical approach consists in restoring the vertebral height, with balloons, after some types of compression fractures, followed by stabilisation using injected bone cement. The kyphoplasty idea came to one of the Kyphon Inc founder Mark A Reiley in 1984 and 15 years laters in 1999, the first kyphoplasty kits were sold with the commercial development, sustained by 40 million dollars in venture capital (http://www.encyclopedia.com/ doc/1G2-2690300069.html. Accessed 15 March 2016). The benefit of kyphoplasty over vertebroplasty was further evidenced by a study analysing the mortality risk of vertebral compression fracture patients $(>800,000)$, registered in the US Medicare data set [11]. Today, with higher clinical evidence requests for market approval, rewriting such a successful and compelling story is most certainly beyond of reach of most medtech companies, except of large companies or alike BASS syndicates of SMEs for the mutualisation of not only the skills, but also the funds.

In response to the requirements of commercial translation, a big growing trend is the formation of clusters of SMEs and large companies around major clinical and university centres (e.g., Karolinska University, UCL London, Maastricht University, Aachen University, NUI Galway's SFI Centre for Research in Medical Devices (CÚRAM)). Some large companies are shifting, therefore, their traditional in-house $R \& D$ to be more present on those campuses (e.g., Stanford Research Park). The real question is still remaining: once you have found something interesting, how to efficiently leverage it?

Limitations of collaboration should also be considered. Promoting open innovation is certainly attractive, but important aspects should not be disregarded, such as intellectual property issues, the implementation of business agreements (e.g., non-disclosure agreement, collaborative research agreement, material transfer agreement) and the share of the potential revenues by all contributing parties to be made from the innovations.

Innovation is becoming more a collaboration with multiple groups (e.g., hospitals, universities, SEMs, large companies) and is becoming essential in the rapidly changing environment. But the world is huge. It is still a matter of communicating or meeting at the right place and at the right time. And then, a lot of water needs to flow under the bridge. 


\section{Compliance with ethical standards}

Conflict of interest The authors declare that they have no competing interests.

\section{References}

1. Holzapfel BM, Reichert JC, Schantz JT, Gbureck U, Rackwitz L, Nöth U, Jakob F, Rudert M, Groll J, Hutmacher DW. How smart do biomaterials need to be? A translational science and clinical point of view. Adv Drug Deliv Rev. 2013;65(4):581-603.

2. Richards RG. AO Research Institute Davos within the AO Foundation: A model for translation of science to the clinics. J Orthop Translation. 2013;1:11-18.

3. ter Boo G-JA, Grijpma DW, Moriarty TE, Richards RG, Eglin D. Antimicrobial delivery systems for local infection prophylaxis in orthopedic- and trauma surgery. Biomaterials. 2015;52:113-25.

4. Kurien $\mathrm{T}$, et al. Bone graft substitutes currently available in orthopaedic practice. The evidence for their use. Bone Joint $\mathrm{J}$. 95-B, (5), 2013.

5. Chatterji AK, Fabrizio KR. Does the market for ideas influence the rate and direction of innovative activity? Evidence from the medical device industry. Strat Mgmt J. 2014; doi:10.1002/ smj.2340.

6. Bayon Y, et al. Progressing innovation in biomaterials. From the bench to the bed of patients. J Mater Sci Mater Med 2015;26:228. doi:10.1007.

7. Mattsson P. Resorbable bone cement for augmentation of hip fracture. Doctoral Thesis University of Uppsala, ISBN: 91-5546271-5, 2005.

8. Kettenberger $\mathrm{U}$, et al. In vitro and in vivo investigation of bisphosphonate loaded hydroxyapatite particles for peri-implant bone augmentation. J Tissue Eng Regen Med. 2015. doi: 10.1002/ term.2094.

9. Landis SC, Amara SG, Asadullah K, Austin CP, Blumenstein R, Bradley EW, Finkelstein R. A call for transparent reporting to optimize the predictive value of preclinical research. Nature. 2012;490(7419):187-91.

10. Chatterji AK. Spawned with a silver spoon? Entrepreneurial performance and innovation in the medical device industry. Strat Mgmt J. 2009;30(2):185-206.

11. Edidin AA, Ong KL, Lau E, Kurtz SM. Mortality risk for operated and nonoperated vertebral fracture patients in the medicare population. J Bone Miner Res. 2011;26(7):1617-26. 